\title{
Escherichia coli enterobactin synthesis and uptake mutants are hypersensitive to an antimicrobial peptide that limits the availability of iron in addition to blocking Holliday junction resolution
}

\author{
Correspondence \\ Anca M. Segall \\ asegall@sunstroke.sdsu.edu
}

Received 30 August 2011

Revised 14 November 2011

Accepted 16 November 2011

\author{
Samantha S. Orchard, † Jason E. Rostron and Anca M. Segall
} Department of Biology and Center for Microbial Studies, San Diego State University,
5500 Campanile Drive, San Diego, CA 92182, USA

\section{INTRODUCTION}

Holliday junctions are DNA structures with four doublestranded arms that serve as intermediates in recombination-dependent repair and rearrangement of DNA in both prokaryotes and eukaryotes. We have identified synthetic hexapeptides that bind Holliday junctions as disulfidebridged dimers and inhibit Holliday junction resolution (Boldt et al., 2004; Kepple et al., 2005). One of these peptides, with the sequence wrwycr (we use the D-amino acid form to limit biological degradation), is a potent, broad-spectrum antimicrobial with the ability to inhibit, in vivo, mechanisms of DNA recombination and damage repair that proceed through a Holliday junction intermediate (Gunderson \& Segall, 2006; Gunderson et al.,

tPresent address: Verenium Corp., 4955 Directors Place, San Diego, CA 92121, USA.

Abbreviations: EPR, electron paramagnetic resonance; qPCR, quantitative PCR; RP-HPLC, reverse-phase HPLC.

Supplementary material, containing extended methods, five figures and three tables, is available with the online version of this paper.
2009; L. Marcusson and other authors, unpublished data). Because mammalian host defences such as gastric acid and the oxidative burst in macrophages induce DNA damage and thus DNA repair in bacterial invaders, use of wrwycr in antimicrobial therapy may increase the efficacy of these natural defence systems. Recently, wrwycr was shown to inhibit the growth of Salmonella enterica serovar Typhimurium inside macrophages (Su et al., 2010) and significantly enhance acid-induced killing of Shiga toxin-producing strains of Escherichia coli associated with haemolytic uraemia syndrome (Lino et al., 2011).

While it is known that wrwycr targets Holliday junctions, it is not known how wrwycr enters cells, what other cellular targets it may have and what defence mechanisms cells employ against wrwycr. To address these questions, we screened a knockout library of $E$. coli for mutants that are resistant or hypersensitive to wrwycr. We expected mutations that conferred wrwycr resistance to inform us of cellular targets of wrwycr or mechanisms of wrwycr entry into the cell. We expected wrwycr-hypersensitive mutants to inform us of wrwycr efflux or other detoxification mechanisms as 
well as pathways that, when perturbed, increase targets for the peptide. From a set of wrwycr-hypersensitive mutants described here, we have discovered that wrwycr also affects the regulation of iron-responsive genes. Incongruously, wrwycr appears to simultaneously increase the measurable amount of desferrioxamine-chelatable iron and decrease the pool of bioavailable iron in the cell. This latter trait makes wrwycr especially detrimental to bacteria in iron-deficient environments or to bacteria that, through mutation, have lost components of their high-affinity iron-uptake system or their iron-responsive regulatory system.

Iron is an essential element for almost all organisms, but can also be toxic. In what is known as Fenton chemistry, iron catalyses the decomposition of hydrogen peroxide (Fenton, 1894), resulting in hydroxyl radical and hydroxyl anion production (Haber \& Willstätter, 1931; Haber \& Weiss, 1932, 1934). The hydroxyl radical product can damage DNA, proteins and lipids (Halliwell \& Gutteridge, 1984; Imlay et al., 1988). Because Fenton reactions are catalysed by free iron, cells regulate the amount of iron they import from their environment. Fur, an ironresponsive transcriptional regulator, plays a key role in this regulation in E. coli by repressing transcription of genes encoding iron-uptake proteins in the presence of sufficient iron (Bagg \& Neilands, 1987). In low-iron conditions, Fur no longer binds to the promoters and transcription of these same genes is derepressed.

An additional layer of iron regulation in E. coli is mediated through the small RNA RyhB, which, as a repressor, acts in concert with the RNA-binding protein $\mathrm{Hfq}$ to facilitate target transcript degradation by RNase E (Massé et al., 2003). While Fur regulates the amount of incoming iron, RyhB aids degradation of certain transcripts to direct available iron away from non-essential uses (such as iron storage or incorporation into non-essential proteins) in low-iron conditions, thus ensuring that the limited amount of iron is put to the most beneficial use (Massé et al., 2005). RyhB has also been shown to increase the levels of some transcripts (Massé et al., 2005), perhaps by preventing their degradation by RNases, and to activate translation of the shiA (shikimate transport) transcript by allowing access to the transcript's ribosome-binding site (Prévost et al., 2007). In high-iron conditions, Fur represses transcription of $r y h B$ (Massé \& Gottesman, 2002; Vassinova \& Kozyrev, 2000), leading to an increase in iron storage and a decrease in shikimate uptake.

Many bacteria, both environmental and pathogenic species, have specialized systems for taking up iron from their environments, which can be very low in soluble iron (Neilands, 1981). Enterobactin is a siderophore synthesized non-ribosomally in a number of Gram-negative bacteria and in a few Gram-positive bacteria (reviewed by Raymond et al., 2003). It is exported to the extracellular milieu by EntS (Furrer et al., 2002) and TolC (Bleuel et al., 2005) and, once it has bound iron to become ferric enterobactin, is imported by the cell (Supplementary Fig. S1, available with the online version of this paper). FepA is an outermembrane protein that transports extracellular ferric enterobactin into the periplasm; the TonB-ExbB-ExbD complex associated with the cytoplasmic membrane provides the energy for the activity of FepA (Guerinot, 1994). FepB binds to ferric enterobactin in the periplasm and directs it to the FepD-FepG cytoplasmic membrane transporter; FepC is an ATPase that assists FepD and FepG in transporting ferric enterobactin into the cytoplasm (Guerinot, 1994). Fes is the ferric enterobactin esterase that degrades ferric enterobactin into three 2,3-dihydroxybenzoyl-L-serine units to release iron, which is then reduced by an unknown mechanism (Brickman \& McIntosh, 1992).

In our screen for E. coli mutants with altered sensitivity to wrwycr, we identified several physiologically related hypersensitive mutants: $\Delta f e p B, \Delta f e p C, \Delta f e p D, \Delta f e p G, \Delta f e s$, $\Delta$ tolC and $\Delta f u r$. In the course of the work presented here, we found that $\Delta e n t B, \Delta e n t C$ and $\Delta m e n F$ mutants were also wrwycr-hypersensitive. Fur represses expression of the fep genes in high-iron conditions, EntB and EntC are involved in synthesizing enterobactin (Supplementary Fig. S1) in addition to other compounds (Bleuel et al., 2005; Koronakis, 2003), and MenF synthesizes isochorismate for menaquinone production (Daruwala et al., 1996). We present evidence that wrwycr limits iron availability (although it actually increases chelatable iron in the cell) or otherwise alters iron regulation in the cell, causing hyper-repression or hypo-activation of an unidentified gene by RyhB and thus hypersensitivity in the fur and enterobactin synthesis and uptake mutants.

\section{METHODS}

Strains, media and chemicals. Strains (Supplementary Table S1, available with the online version of this paper) were routinely cultured in $\mathrm{LB}\left[5 \mathrm{~g} \mathrm{NaCl}^{-1}\right.$ (Davis et al., 1980)] at $37{ }^{\circ} \mathrm{C}$ with shaking (225 r.p.m.). The medium used for screening the Keio collection, no citrate E salts (NCE)/glucose/Casamino acids/vitamins (NGCV), was $1 \times$ NCE minimal medium base (Davis et al., 1980) with $0.2 \%$ glucose, $1 \%$ autoclaved Casamino acids and $1 \times$ vitamin mix $1(500 \times$ stock of vitamin mix 1 is an autoclaved mixture of $0.025 \%$ folic acid, $0.025 \%$ pantothenic acid, $0.025 \%$ nicotinamide, $0.025 \%$ pyridoxal- $\mathrm{HCl}, 0.025 \%$ thiamine- $\mathrm{HCl}, 0.0025 \%$ riboflavin and $0.05 \%$ biotin) and no added iron. The MOPS/glucose/Casamino acids/vitamins (MGCV) medium was based on a component of a 'culture medium for enterobacteria' (Neidhardt et al., 1974) and consisted of $40 \mathrm{mM}$ MOPS, $4 \mathrm{mM}$ Tricine (the $10 \times$ MOPS/Tricine mixture was adjusted to $\mathrm{pH} 7.4$ with $\mathrm{KOH}), 9.5 \mathrm{mM} \mathrm{NH}_{4} \mathrm{Cl}$, $0.276 \mathrm{mM} \mathrm{K}_{2} \mathrm{SO}_{4}, 0.5 \mu \mathrm{M} \mathrm{CaCl}, 0.525 \mathrm{mM} \mathrm{MgCl}_{2}, 5 \mathrm{mM} \mathrm{NaCl}$, $1.32 \mathrm{mM} \mathrm{K} \mathrm{HPO}_{4}, 0.2 \%$ glucose, $1 \%$ Casamino acids and $1 \times$ vitamin mix $2(1000 \times$ stock of vitamin mix 2 is a filter-sterilized mixture of $0.05 \%$ folic acid, $0.05 \%$ pantothenic acid, $0.05 \%$ nicotinamide, $0.05 \%$ nicotinic acid, $0.05 \%$ pyridoxine- $\mathrm{HCl}, 0.05 \%$ thiamine- $\mathrm{HCl}, 0.005 \%$ riboflavin and $0.1 \%$ biotin, with $\mathrm{NaOH}$ added to assist solubilization). $\mathrm{FeCl}_{3}$ was added to 2.4 or $6 \mu \mathrm{M}$ (as indicated) for low-iron conditions and up to $150 \mu \mathrm{M}$ (as indicated) for high-iron conditions. Mueller-Hinton broth powder was commercially prepared (Becton Dickinson). Chemicals and media components were purchased from Sigma-Aldrich or Fisher Scientific unless indicated otherwise. The peptide wrwycr consisted of D-amino 
acid residues with an amide group at the C-terminus, and was synthesized and purified to $>95 \%$ purity by BioSynthesis or SigmaGenosys. See Supplementary Material for full details on D-wrwycr.

Screening of Keio collection for hypersensitive mutants. To screen the Keio collection of 3985 mutants (Baba et al., 2006), a 96place replicator was used to inoculate microtitre wells containing the tolerance-testing medium $(100 \mu \mathrm{l})$ with overnight cultures. The tolerance-testing medium was NGCV with either DMSO (solventonly control; final concentration $0.5 \%)$ or wrwycr $(20 \mu \mathrm{M}$ with $0.2 \%$ final DMSO concentration) added. The inoculated plates were sealed with paraffin film to reduce evaporation, incubated at $30{ }^{\circ} \mathrm{C}$ and the $\mathrm{OD}_{600}$ was read at 24 and $72 \mathrm{~h}$. To factor out the different wrwycrfree growth abilities of the various mutant strains, the optical density reading in the presence of wrwycr for each time point was divided by the optical density for the same strain in the presence of DMSO alone to get a ratio. Typically, mutants with a ratio of $<0.3$ were deemed to be candidate hypersensitive strains if the majority of strains on the plate had a higher ratio. For verified hypersensitive strains and additional Keio collection strains of interest, the mutated locus was transduced into E. coli MG1655 (Supplementary Table S1) or another desired genetic background using bacteriophage P1 (Miller, 1972). Where indicated, the kanamycin-resistance cassette (FRT-kan ${ }^{\mathrm{r}}$-FRT) was removed via recombination between the flanking FRT sites using Flp recombinase supplied from plasmid pCP20 (Cherepanov \& Wackernagel, 1995); the strains were subsequently cured of pCP20 by growth at $42{ }^{\circ} \mathrm{C}$. The deletion/insertion site of all listed strains (Supplementary Table S1) was verified by PCR.

Other strain construction. The $\Delta r y h B$ mutant strain was constructed by replacing the $r h y B$ locus with a FRT-kan ${ }^{\mathrm{r}}$-FRT cassette from plasmid pKD13 into strain EDT1160 and transducing the $\Delta r y h B::$ FRT-kan ${ }^{\mathrm{r}}$-FRT locus into strain G652 to create strain EDT1571. The $\Delta$ fep/fes $\Delta r y h B$ double mutants were constructed by transducing the $\Delta r y h B::$ FRT-kan ${ }^{\mathrm{r}}$-FRT locus into the appropriate single mutants (Supplementary Table S1) using P1. To create strain EDT1689, the FRT-kan ${ }^{\mathrm{r}}$-FRT cassette was removed from EDT1571; to create strain EDT1712, the $\Delta$ fur::FRT-kan ${ }^{\mathrm{r}}$-FRT locus was transduced into strain EDT1689. Full details of these methods can be found in the Supplementary Material.

Growth assays. Because phosphate, a major component of NCE and thus the NGCV medium, co-precipitates with iron and other cations, a MOPS-based medium (MGCV, above) was used for growth studies involving the addition of iron, except when noted. For wrwycr sensitivity and iron complementation assays, overnight cultures were diluted to $1 \%$ in MGCV, or NGCV for the $\Delta$ fur and $\Delta$ fur $\Delta r y h B$ mutants, containing the concentration of wrwycr indicated in figure legends or the same volume of $100 \% \mathrm{DMSO}$, and $\mathrm{FeCl}_{3}$ (dissolved in $0.1 \mathrm{M} \mathrm{HCl}$ ) added at the concentrations indicated in the figure legends or the same volume of $0.1 \mathrm{M} \mathrm{HCl}$ in a 96-well plate; the plates were sealed with paraffin film, grown at $37{ }^{\circ} \mathrm{C}$ with 1 min agitation every $10 \mathrm{~min}$, and the $\mathrm{OD}_{600}$ was periodically assayed in a VersaMax or SpectraMax plate reader (Molecular Devices). The data are presented with the $y$-axis on a $\log _{2}$ scale, as appropriate for cell growth data.

Determining dimer : monomer ratio and cell-associated concentration of wrwycr. Reverse-phase (RP)-HPLC was used to visualize (by absorbance at $280 \mathrm{~nm}$ ) and separate the monomer and dimer peaks of wrwycr in order to monitor the extent of dimerization of the wrwycr stocks and to determine the amount of cell-associated wrwycr in formic acid cell extracts.

For determining dimer : monomer in stock solutions, wrwycr diluted in $\mathrm{H}_{2} \mathrm{O}$ was applied to a C8-on-silica column (Jupiter $4 \mu \mathrm{M}$ Proteo Column $90 \AA$, Phenomenex) in an $\mathrm{H}_{2} \mathrm{O} / 0.1 \%$ trifluoroacetic acid mobile phase, then eluted with a segmented linear gradient of acetonitrile $/ 0.1 \%$ trifluoroacetic acid with a flow rate of $1 \mathrm{ml} \mathrm{min}{ }^{-1}$. To determine the cell-associated concentration of wrwycr, overnight cultures in MGCV with $6 \mu \mathrm{M} \mathrm{FeCl}_{3}$ were diluted to $1 \%$ in MGCV with $6 \mu \mathrm{M} \mathrm{FeCl}_{3}$ and incubated with shaking at $37^{\circ} \mathrm{C}$ for $1.5 \mathrm{~h}$ before wrwycr was added to $10 \mu \mathrm{M}$. After a further $1.5 \mathrm{~h}$ incubation, the $\mathrm{OD}_{600}$ was measured and $1 \mathrm{ml}$ of the culture was layered onto $500 \mu \mathrm{l}$ of a 1:1 mix of AR20 and AP100 silicone oils, which was itself layered over a $70 \mu \mathrm{l}$ bed of $20 \%$ formic acid in a $1.7 \mathrm{ml}$ microcentrifuge tube (protocol adapted from an unpublished protocol by E. Ozyamak and I. Booth, University of Aberdeen, Scotland). Following centrifugation, $35 \mu \mathrm{l}$ of the formic acid solution of each sample was mixed with $215 \mu \mathrm{l}$ water and $100 \mu \mathrm{l}$ of the mix was analysed by RP-HPLC as described above. The area under the monomer and dimer wrwycr peaks was used to determine the relative amount of wrwycr in each sample. Bacteria not treated with wrwycr were treated and analysed similarly. The RP-HPLC results were compared with those of formic acid only to identify a cell-dependent peak that eluted from the column at approximately 6-7 min, the area of which correlated with the number of untreated cells used in the extraction up to a maximum of $\sim 109$ cells.

The area under the cell-dependent peak or the $\mathrm{OD}_{600}$ of the treated cultures was used to normalize the results for total wrwycr concentration to account for differences in the number of sampled cells. Data for each normalization method are shown separately. Full details are given in the Supplementary Material.

Quantitative PCR (qPCR) of Fur regulon genes. Overnight cultures $(200 \mu \mathrm{l})$ from three independent colonies per strain grown in MHB were used to inoculate $20 \mathrm{ml}$ MHB in $50 \mathrm{ml}$ flasks. After $1 \mathrm{~h}$ growth, $10 \mu \mathrm{M}$ wrwycr or $0.1 \%$ DMSO was added to the cultures. After $1.5 \mathrm{~h}$ further growth, RNA was extracted and used to synthesize cDNA, which was then analysed by qPCR. The qPCR conditions are given in the Supplementary Material and the primers used are listed in Supplementary Table S2.

4-Methylumbelliferyl- $\beta$-D-glucuronide (MUG) assays for $\beta$-galactosidase activity. Overnight cultures in MGCV supplemented with $6 \mu \mathrm{M} \mathrm{FeCl}_{3}$ were subcultured 1:500 in fresh $\mathrm{MGCV}+6 \mu \mathrm{M} \mathrm{FeCl}_{3}$ and incubated at $37{ }^{\circ} \mathrm{C}$ until the cultures reached $\mathrm{OD}_{600} \sim 0.3$. Cells were treated in a 96-well microtitre plate by adding $100 \mu \mathrm{l}$ of the cultures to $100 \mu \mathrm{MGCV}+6 \mu \mathrm{M} \mathrm{FeCl}_{3}$ containing twice the indicated concentration of wrwycr. After lysis, $100 \mu \mathrm{l} 1 \times$ buffer $\left(60 \mathrm{mM} \mathrm{K} \mathrm{KPO}_{4}\right.$, $40 \mathrm{mM} \mathrm{KH}_{2} \mathrm{PO}_{4}, 100 \mathrm{mM} \mathrm{NaCl}$ ) was added to the aliquots, followed by $10 \mu \mathrm{l}$ MUG $\left(0.4 \mathrm{mg} \mathrm{ml}^{-1}\right)$. The plate was placed in the dark for $45 \mathrm{~min}$ and the reaction was stopped by the addition of $100 \mu \mathrm{l}$ glycine buffer $(200 \mathrm{mM}, \mathrm{pH} 10.3)$. The fluorescence intensity of the product, 4-methylumbelliferone, was measured at an excitation of $360 \mathrm{~nm}$ and an emission of $450 \mathrm{~nm}$ in a Molecular Devices SpectraMax GEMINI plate reader (Molecular Devices). The fluorescence was normalized by the $\mathrm{OD}_{600}$ of the culture at the time the $10 \mu \mathrm{l}$ aliquots were taken. See Supplementary Material for full details.

Catalase assays Catalase activity in extracts of various bacterial strains was determined using an assay described previously ( $\mathrm{Li} \&$ Schellhorn, 2007). Briefly, MG1655 and the $\Delta f e p B$ strain were grown for $1.5 \mathrm{~h}$, then DMSO or wrwycr (to $10 \mu \mathrm{M}$ ) was added to the culture and cells were sampled after a further 1.5 or $3 \mathrm{~h}$ incubation. Cell pellets from overnight or treated cultures were resuspended in $50 \mathrm{mM}$ potassium phosphate buffer, $\mathrm{pH} 7.0$, lysed by sonication and the cell debris was removed by centrifugation. Total protein concentration in the cell extracts was determined by using a Bradford-type assay (BioRad). Immediately before assay readings were taken, $250 \mu \mathrm{l} 5 \mathrm{mM}$ $\mathrm{H}_{2} \mathrm{O}_{2}$ in $50 \mathrm{mM}$ potassium phosphate buffer, $\mathrm{pH} 7.0$, was added to samples and standards in a 96-well UV-transparent plate (Costar 3635, Corning). Catalase activity was assayed by monitoring 
absorbance at $240 \mathrm{~nm}$, which decreases as $\mathrm{H}_{2} \mathrm{O}_{2}$ is degraded. The $A_{240}$ was recorded in a SpectraMax plate reader (Molecular Devices) for $5 \mathrm{~min}$ at $\sim 8 \mathrm{~s}$ intervals, the slope of change in $A_{240}$ (calculated for each time point relative to $t=0$ ) was determined, and catalase activity (in units per mg total protein) was calculated from the standard curve and protein concentration determination. Full details are given in the Supplementary Material.

Electron paramagnetic resonance (EPR) spectroscopy. Intracellular chelatable iron concentrations were measured by EPR spectroscopy as described by Woodmansee \& Imlay (2002). Full details are given in the Supplementary Material.

Determination of mutation frequency using the median method. Sixteen overnight cultures, each inoculated from an independent colony, were subcultured 1:500 in NGCV media with no added iron and grown to $\mathrm{OD}_{600} 0.15$, then diluted $1: 1$ with an equal volume of NGCV medium containing either DMSO alone or wrwycr to achieve a final concentration of 8 or $16 \mu \mathrm{M}$. Aliquots were taken at time 0 to determine the number of pre-existing mutants, as well as after 3 or $24 \mathrm{~h}$ treatment. No cultures with pre-existing mutations were used in this determination. Aliquots $(2.5 \mu \mathrm{l})$ of $10-$ fold serial dilutions $\left(10^{-1}-10^{-6}\right)$ were spot-plated on LB agar without any additions to determine the viable count. The remaining $190 \mu \mathrm{l}$ was spread onto LB agar plates supplemented with $50 \mu$ g rifampicin $\mathrm{ml}^{-1}$. The number of large rifampicin-resistant colonies was counted and the median number of mutants from all the cultures was divided by the mean total colonies at each time point to obtain the mutation frequency.

Statistical analysis. A one-way analysis of variance with Tukey's multiple comparison test was performed to compare the total amount and dimer : monomer ratio of cell-associated wrwycr detected by RPHPLC for all samples. A two-tailed $t$-test was performed to analyse the qPCR and catalase results (Prism version 5.0b for Macintosh; GraphPad Software).

\section{RESULTS}

\section{Identification of wrwycr-hypersensitive E. coli mutants}

The Keio collection of E. coli deletion-insertion mutants was partially screened to find mutations that increased or decreased the tolerance of $E$. coli for the peptide wrwycr (Methods). Here we describe our investigation of a subset of strains that appeared hypersensitive in this screen, a group of mutants related to enterobactin synthesis and transport, namely $\Delta f e p B, \Delta f e p C, \Delta f e p D, \Delta f e p G$ and $\Delta f e s$. FepB (a periplasmic binding protein), FepC, FepD and FepG are involved in the uptake of the iron-bound siderophore molecule ferric enterobactin across the cytoplasmic membrane, and Fes breaks down imported ferric enterobactin in the cytoplasm to release iron (Guerinot, 1994). We verified the hypersensitivity of these mutants by transducing the mutations into E. coli MG1655 and testing the response of the MG1655-based strains to wrwycr compared with that of the MG1655 parent strain. We also measured the effect of two other ferric enterobactin uptake-related mutations transduced from the Keio collection, $\Delta$ fepA and $\Delta t o n B$, on sensitivity of MG1655 to wrwycr. We found that, in the MG1655 background,
$\Delta f e p B, \Delta f e p C, \Delta f e p D, \Delta f e p G, \Delta$ tonB and $\Delta f e s$ showed no growth $\left(\mathrm{OD}_{600}\right.$ ratio $\left.<0.3\right)$ at $30{ }^{\circ} \mathrm{C}$ in $15 \mu \mathrm{M}$ wrwycr in NGCV medium, which has no added iron other than that introduced by other media constituents or from the glassware, even after $72 \mathrm{~h}$ incubation. The $\Delta$ fep $A$ mutant showed hypersensitivity only at the $24 \mathrm{~h}$ and not at the 48 and $72 \mathrm{~h}$ observation points. MG1655 is resistant $\left(\mathrm{OD}_{600}\right.$ ratio $>0.8$ ) to $15 \mu \mathrm{M}$ wrwycr in the same conditions (for comparison, it is sensitive to $25-30 \mu \mathrm{M}$ wrwycr). We removed the kanamycin resistance cassette from the MG1655-based strains and followed their growth when challenged with $22 \mu \mathrm{M}$ wrwycr in MOPS-based MGCV medium (Methods) containing $2.4 \mu \mathrm{M} \mathrm{FeCl}_{3}$; this amount of iron was chosen because the enterobactin uptake mutants were unable to grow in lower concentrations of $\mathrm{FeCl}_{3}$ in MGCV in the absence of wrwycr. This medium contains a low level of phosphate, insufficient to precipitate the added iron, unlike the greater amount of phosphate present in NGCV. All the mutants grew essentially the same as the parent strain in $\mathrm{MGCV} / 2.4 \mu \mathrm{M} \mathrm{FeCl}$ sithout wrwycr but with the DMSO solvent (Fig. 1a). In contrast, the growth of the mutants, except $\triangle f e p A$ and $\triangle t o n B$, in MGCV/2.4 $\mu \mathrm{M} \mathrm{FeCl}_{3}$ with wrwycr was delayed relative to M1655 (open symbols in Fig. 1b, c), verifying the original phenotype; the $\triangle f e p A$ and $\Delta t o n B$ mutants showed very little hypersensitivity to wrwycr in this assay (Fig. 1b). In all cases, the mutants recovered, often with a similar growth rate; we show below that this recovery is likely to be largely dependent on the TolC-dependent efflux, since the effluxdefective $\Delta t o l C$ strain is hypersensitive to wrwycr and loses much of its ability to recover.

We reasoned that if wrwycr hypersensitivity in the $\Delta f e p$ and $\Delta$ fes mutants was caused by hindrance of ferric enterobactin transport across the cytoplasmic membrane rather than hindrance of transport of another compound through the same system, the impairment of enterobactin synthesis would also render E. coli hypersensitive to wrwycr. We thus transduced the mutated loci of ent $B$ and ent $C$, two genes whose products are required for enterobactin synthesis, from the corresponding Keio collection strains to the MG1655 background and measured the growth of the resulting strains in wrwycr. While both the enterobactin synthesis mutants tested were hypersensitive to wrwycr, the $\triangle e n t B$ strain was more hypersensitive than the $\Delta e n t C$ mutant strain (Fig. 1c). These results suggest that enterobactin synthesis is required for wild-type-level resistance to wrwycr but that there is an EntC-independent way of making or taking up the dihydroxybenzoate substrate for enterobactin synthesis under our test conditions. EntC is an isochorismate synthase and E. coli encodes another isochorismate synthase, MenF (Daruwala et al., 1996), which is needed to produce menaquinone for electron transport in anaerobic conditions. As our growth assays were done in sealed, predominantly static 96 -well microtitre plates with glucose as the carbon source, it is likely that the dissolved oxygen content in the cultures was very low. Therefore, we tested whether MenF could substitute for EntC in 

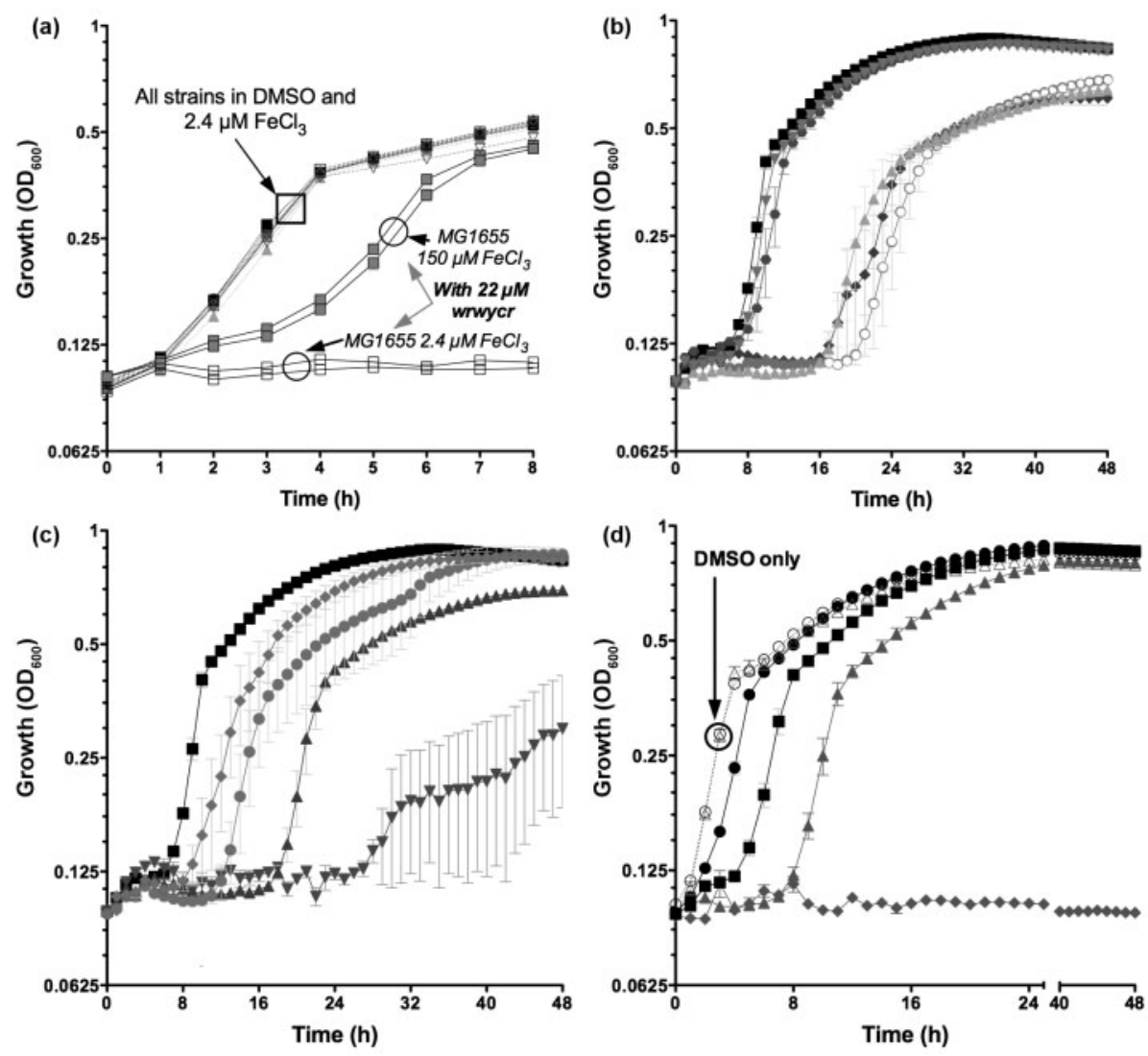

Fig. 1. E. coli enterobactin synthesis, export and uptake mutants in MGCV medium are hypersensitive to wrwycr. (a) The mutants grow similarly to the parent (MG1655) in DMSO (dotted lines) in 'low'-iron conditions (2.4 $\mu \mathrm{M} \mathrm{FeCl}_{3}$ added), as indicated by a box. For comparison purposes, the $\mathrm{OD}_{600}$ of MG1655 (G652) grown in $22 \mu \mathrm{M}$ wrwycr and either $150 \mu \mathrm{M} \mathrm{FeCl}{ }_{3}$ ( $\square$ ) or $2.4 \mu \mathrm{M} \mathrm{FeCl}_{3}(\square)$ is also shown. Because the strains were split into two plates for this analysis, the data for the two separate sets of MG1655 comparison wells on each plate are shown. (b-d) Four individual colonies of (b) MG1655 (G652, 口), $\Delta f e p B(E D T 1343, \nabla), \Delta f e p D(E D T 1344,0), \Delta f e s(E D T 1345, \Delta), \Delta f e p A(E D T 1574, \bigcirc)$, and $\Delta$ tonB (EDT1688, V) or (c)

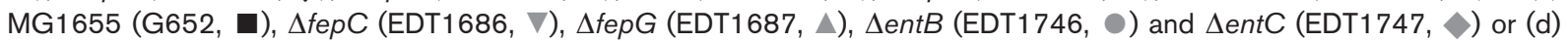
MG1655 (G652, $\bullet$ and $\mathbf{\square})$ and $\Delta$ tolC (EDT1350, $\Delta$ and $\diamond)$ were grown overnight in MGCV medium $\left(2.4 \mu \mathrm{M} \mathrm{FeCl}_{3}\right)$, then diluted to $1 \%$ in fresh MGCV medium with $22 \mu \mathrm{M}$ wrwycr (all data in b and c), $5 \mu \mathrm{M}$ wrwycr (circles and triangles in d), $10 \mu \mathrm{M}$ wrwycr (squares and diamonds in d) or DMSO (dashed lines and open symbols in d) and $2.4 \mu \mathrm{M} \mathrm{FeCl}_{3}$. The cultures (100 $\mu \mathrm{l}$ total) were grown in microtitre plates at $37{ }^{\circ} \mathrm{C}$. Each data point represents the mean $\pm S E M \mathrm{OD}_{600}$ of the four individual cultures tested. Growth measurements shown in (b) and (c) were recorded simultaneously but are presented in two panels for clarity.

enterobactin synthesis in the low-oxygen environment of microtitre plates. A $\triangle m e n F$ mutant had a hypersensitivity to wrwycr intermediate between that of the wild-type strain and the $\triangle$ entC mutant strain (Supplementary Fig. S2a), while a $\Delta e n t C \Delta m e n F$ double mutant was more sensitive to wrwycr than either of the single mutants (Supplementary Fig. S2a), suggesting that either MenF activity can compensate for the lack of EntC for enterobactin synthesis in our assay conditions or menaquinone synthesis itself is important for resistance to wrwycr in low-oxygen environments.

\section{A $\Delta t$ tolC mutant is hypersensitive to wrwycr}

Another wrwycr-hypersensitive strain identified in the original screen was the $\Delta t o l C$ mutant. This strain was significantly more sensitive to wrwycr than were the other strains reported here; it did not grow in $10 \mu \mathrm{M}$ wrwycr even after $48 \mathrm{~h}$ (Fig. 1d), while the other mutants all grew at least somewhat in $22 \mu \mathrm{M}$ wrwycr (Figs $1 \mathrm{~b}$ and 2c). TolC not only is essential for the export of newly synthesized enterobactin across the outer membrane (Bleuel et al., 2005) but also assists in the efflux of many other compounds, including antibacterial drugs (reviewed by Koronakis et al., 2004). EntS is involved in transport of enterobactin across the cytoplasmic membrane (Furrer et al., 2002) and thus an entS mutant could be expected to have the same phenotype as a tolC mutant. However, the E. coli $\Delta e n t S$ strain was not hypersensitive to wrwycr (Supplementary Fig. S2b). This suggests that enterobactin export by EntS is not essential for the ability 

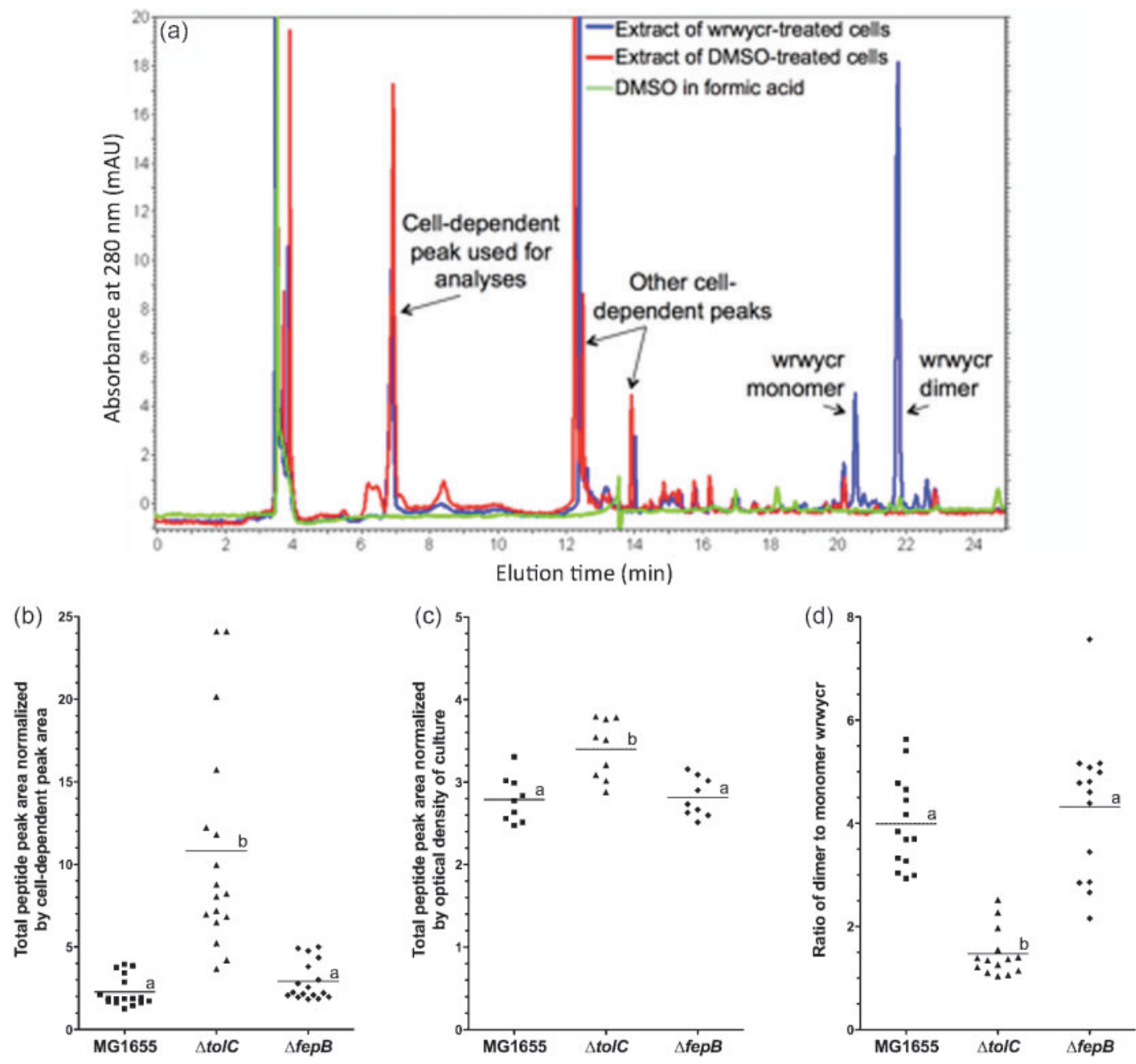

Fig. 2. The E. coli $\Delta t o / C$ mutant, but not the $\Delta f e p B$ mutant, has an increased level of cell-associated wrwycr and a lower ratio of dimer to monomer wrwycr relative to the MG1655 parent strain, as measured in an RP-HPLC assay. (a) Overlay of typical RPHPLC traces of formic acid extracts from E. coli MG1655 (G652) treated with wrwycr (solid line) or DMSO (short-dashed line) and of DMSO in formic acid (long-dashed line) to show identification of cell-dependent peaks and monomer and dimer wrwycr, as indicated. (b-d) Exponential-phase MG1655 (G652, $\mathbf{a}), \Delta$ to/C (EDT1311, $\mathbf{\Delta})$ or $\Delta$ fepB (EDT1316, $\boldsymbol{\Delta})$ cells were treated with $10 \mu \mathrm{M}$ wrwycr for $1.5 \mathrm{~h}$, after which cell-associated peptides were extracted using formic acid. The formic acid extracts were analysed by RP-HPLC and the detected monomer and dimer wrwycr peak areas were normalized to (b) the area of a celldependent peak, the area of which correlated with the number of DMSO-treated cells subjected to formic acid extraction (data not shown), or to (c) the $\mathrm{OD}_{600}$ of the cultures at the time of formic acid extraction. (b, c) The normalized total peptide amounts expressed as monomer equivalents (b, c) and dimer to monomer ratios (d) are shown. For (b-d), each data point represents the result for an independent culture and the horizontal lines indicate the means of the samples. Data points are from (b) six, (c) three or (d) five separate experiments with three independent cultures each. A one-way analysis of variance with Tukey's multiple comparison test determined that the difference in the means (horizontal bars) within each panel (b-d) is statistically significant $(P>0.05)$ in bars labelled with a different lower-case letter, but not in those labelled with the same letter.

of $E$. coli to withstand wrwycr treatment, probably because entS mutants can export components of enterobactin that are themselves siderophores (Furrer et al., 2002), and that the tolC mutant must have an additional defect that made it hypersensitive to wrwycr. Below, we present evidence that the additional defect is a diminished capacity to export wrwycr.

\section{Added iron reduces the antimicrobial effects of wrwycr by causing its precipitation}

The enterobactin synthesis and uptake mutants described above are deficient in the cell's high-affinity iron- (as ferric enterobactin) uptake system. Our initial screening medium, NGCV, had no added iron and the phosphate buffer 
in the medium may have precipitated any inherent iron. Indeed, the addition of iron to NGCV improves the growth of the wild-type strain in the absence of wrwycr (data not shown). We thus hypothesized that the hypersensitivity of the enterobactin synthesis, export and uptake mutants to wrwycr may be due to their limited ability to get enough iron through their other, lower-affinity iron- uptake systems (for a review see Guerinot, 1994). To address this hypothesis, we added exogenous iron to a low-phosphate, low-iron growth medium, MGCV, and found that the mutants were better able to withstand wrwycr treatment, shown by shorter lag times in the presence of wrwycr (Supplementary Fig. S3). However, we subsequently found that high concentrations of iron $(150 \mu \mathrm{M}$ and above) precipitate the peptide in the media (Supplementary Table S3), thereby lowering the effective concentration of wrwycr to which cells are exposed (Supplementary Fig. S4). Note that we did not observe any significant growth differences between cultures supplemented with $\mathrm{FeSO}_{4}$ versus $\mathrm{FeCl}_{3}$, either at low $(6$ or $12 \mu \mathrm{M})$ or at high $(120 \mu \mathrm{M})$ final concentration (data not shown).

\section{The $\Delta t o / C$ mutant, but not the $\Delta f e p B$ mutant, has an increased level of cell-associated wrwycr relative to the MG1655 parent}

Because the entS mutant was not hypersensitive to wrwycr, we hypothesized that the $\Delta$ tolC mutant is wrwycr-hypersensitive not because of its involvement in enterobactin export but rather because of its inability to efflux wrwycr. To investigate if the tolC mutant is defective for wrwycr efflux, we measured the levels of cell-associated wrwycr in the $\Delta$ tolC mutant and in the parent (MG1655) strains using RP-HPLC (Fig. 2a). 'Cell-associated wrwycr' refers to all the wrwycr that could be extracted by formic acid from the cells, be it in the cytoplasm, periplasm, chromosome or the membranes. We found that the $\Delta t o l C$ mutant does accumulate wrwycr to a higher level than the $\mathrm{TolC}^{+}$MG1655 parent strain (Supplementary Fig. S2b, c), suggesting that the $\Delta t o l C$ mutant is hypersensitive to wrwycr at least in part due to reduced efflux of wrwycr. In contrast, the $\Delta f e p B$ mutant's hypersensitivity is not due to increased cell-associated wrwycr. Interestingly, the $\Delta t o l C$ mutant also showed an increased level of monomer wrwycr relative to dimerized wrwycr (Fig. 2d), while the $\Delta f e p B$ mutant has about the same concentration and dimer-to-monomer ratio of cell-associated wrwycr as MG1655 (Fig. 2b-d).

\section{Transcription of Fur-repressed genes is upregulated in wild-type $E$. coli in response to wrwycr treatment}

The hypersensitivity of the Fep system mutants to wrwycr suggested the possibility that wrwycr restricts the availability of iron to the bacteria. To determine the availability of intracellular iron during wrwycr treatment using a biological assay, we monitored the expression level of two genes, ent $B$ and $f h u F$, that are repressed in high-iron conditions by Fur. Compared to the expression level in MG1655 treated with DMSO, the expression level of entB and $f h u F$ in MG1655 treated with $10 \mu \mathrm{M}$ wrwycr for $1.5 \mathrm{~h}$ was increased by 111 and 161-fold, respectively, on average, as measured by qPCR (Fig. 3). This increased level of expression was nearly as high as that seen in a $\Delta$ fur mutant not treated with wrwycr, in which $f h u F$ and entB expression should be completely derepressed (Fig. 3). This suggests that wrwycr treatment
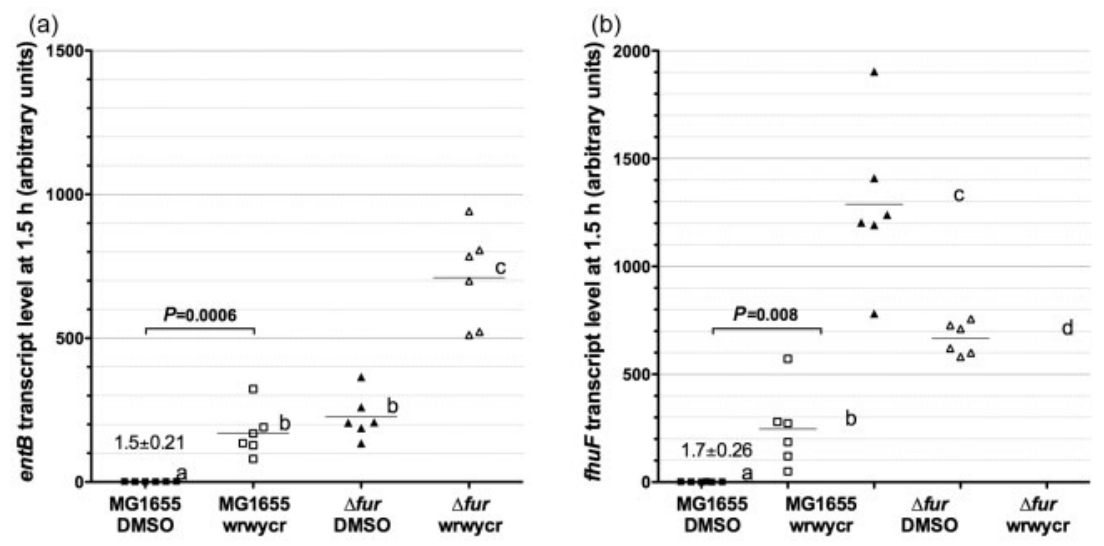

Fig. 3. Quantification of relative transcript levels of (a) entB and (b) fhuF in $E$. coli MG1655 (G652, squares) or $\Delta$ fur (EDT1566, triangles) treated with $0.1 \%$ DMSO ( $(\mathbf{)})$, $10 \mu \mathrm{M}$ wrwycr $(\square), \quad 0.082-0.09 \%$ DMSO $(\Delta)$ or $8.2-9 \mu \mathrm{M}$ wrwycr $(\triangle)$ for $1.5 \mathrm{~h}$ in MHB. Total cellular RNA was harvested from the cultures at $1.5 \mathrm{~h}$, used as a template for cDNA, the cDNA was used in a qPCR, and the results for each sample were adjusted according to the amount of polA cDNA detected for that sample. Horizontal bars indicate the mean of the results for six independent cultures, which were treated and harvested over two separate days (three on each day), and numbers above the bars for MG1655 treated with DMSO provide the mean \pm SEM for those samples. Two-tailed $t$-tests indicated that the difference in the means with different lowercase letters in each panel is statistically significant $(P<0.05$; specific $P$-values are provided in each panel for the comparison of MG1655 treated with DMSO or wrwycr). 
reduces the amount of bioavailable iron in E. coli MG1655 and that the Fur regulon responds accordingly.

We anticipated that entB and $f h u F$ transcript levels in the $\Delta$ fur strain would appear insensitive to wrwycr treatment compared to DMSO treatment. Instead, we did see changes (albeit relatively small, just over threefold), but entB and $f h u F$ transcript levels responded in opposite directions. For example, while the level of $f h u F$ transcripts in wrwycrtreated $\Delta$ fur cultures was about half of that in DMSOtreated $\Delta$ fur cultures at $1.5 \mathrm{~h}$, the level of entB transcripts was about threefold higher for the same comparison. The $\Delta$ fur strain is itself hypersensitive to wrwycr (see below); however, we do not know why wrwycr-treated $\Delta$ fur cultures had different levels of entB and $f h u F$ transcripts from DMSO-treated $\Delta$ fur cultures, or the reason for the differential response of ent $B$ vs $f h u F$ transcripts in the $\Delta f u r$ background (see Discussion).

\section{Growth in NGCV medium and wrwycr treatment decrease catalase activity in cells}

Low bioavailable iron levels lead to a decrease in the cellular concentration of the enzymically active form of non-essential iron-containing enzymes, such as the catalase hydroperoxidase I (KatG or haemoprotein b-590) (Hubbard et al., 1986); thus, catalase activity may indicate iron availability in the cell. Using a $\mathrm{H}_{2} \mathrm{O}_{2}$ depletion assay, we quantified the amount of total catalase activity in cell-free extracts of the enterobactin uptake mutant $\triangle f e p B$ and in the MG1655 parent strain after overnight growth in the screening medium NGCV. Consistent with reduced levels of available iron in the conditions of our screen, we found that extracts of $\Delta f e p B$ grown in NGCV overnight without wrwycr treatment had $29 \%$ of the catalase activity of the parent strain MG1655 in the same conditions (Supplementary Fig. S5a). Treatment of MG1655 in MGCV medium with $10 \mu \mathrm{M}$ wrwycr for $1.5 \mathrm{~h}$ resulted in only $15 \%$ of the catalase activity of the DMSOtreated samples (Supplementary Fig. S5b).

\section{wrwycr treatment increases the chelatable iron in the cell}

The pattern of expression of Fur-regulated genes, observed by qPCR, and the decrease in catalase activity suggested that bioavailable iron levels in the cell are reduced by wrwycr treatment. We used EPR spectroscopy to measure the amount of desferrioxamine-chelatable iron in wrwycr-treated cells. Surprisingly, EPR spectroscopy revealed that levels of chelatable iron in the cell actually increased by six- or sevenfold after 50 min of treatment with 8 or $16 \mu \mathrm{M}$ wrwycr, respectively, relative to cells treated with the same volume of DMSO (Table 1). Combined with our other findings, this suggests that while wrwycr treatment increases chelatable iron in the cell, this iron is not available to Fur or KatG and that additional iron must be imported to rescue cells from the effects of wrwycr.

A possible source of the excess chelatable iron in wrwycrtreated cells may be wrwycr-related damage to proteins
Table 1. Intracellular concentrations of chelatable iron after treatment of MG1655 with wrwycr, measured by EPR spectroscopy

\begin{tabular}{|lcc|}
\hline Treatment & $\begin{array}{c}\text { Chelatable } \\
\text { intracellular } \\
\text { iron }(\boldsymbol{\mu M})^{*}\end{array}$ & $\begin{array}{c}\text { Fold difference } \\
\text { with respect to } \\
\text { DMSO-treated cells }\end{array}$ \\
\hline DMSO & $34.2 \pm 5.5$ & 1 \\
$8 \mu \mathrm{M}$ wrwycr & $201.7 \pm 17.2$ & 5.9 \\
$16 \mu \mathrm{M}$ wrwycr & $238.2 \pm 31$ & 7 \\
\hline
\end{tabular}

${ }^{\star}$ Mean \pm SEM.

that contain iron-sulfur clusters. Kohanski et al. (2007) have suggested that many antibiotics may damage such proteins, causing them to release iron into the cell. We explored this possibility by testing the LacZ levels expressed from the suf operon promoter, which encodes proteins involved in iron-sulfur cluster assembly under ironlimiting or oxidative stress conditions (Outten et al., 2004). Indeed we observed dose-dependent induction of $\beta$ galactosidase activity, which decreased over time at the lower doses of wrwycr tested (Fig. 4a). The suf promoter is activated independently by apo-IscR and OxyR (Yeo et al., 2006). We found that deletion of iscR prevented the rise in wrwycr-dependent LacZ activity, whereas deletion of $\operatorname{oxy} R$ did not affect induction (Fig. 4b). This suggests that ironsulfur clusters are being damaged and are unavailable to bind to apo-IscR following wrwycr treatment.

Free intracellular iron in conjunction with oxygen has been linked to increased DNA damage, leading to higher mutation frequencies (Imlay et al., 1988). To determine if the additional chelatable iron in wrwycr-treated cells leads to an increased DNA mutation frequency, we treated cultures with either 8 or $16 \mu \mathrm{M}$ wrwycr for 3 or $24 \mathrm{~h}$ and measured the mutation frequency compared with that in untreated cultures. To minimize the effect of exogenous iron, cultures were grown in NGCV without any added iron. After $3 \mathrm{~h}$, there was no difference in mutation frequency in treated versus untreated cultures. After $24 \mathrm{~h}$ of treatment, the mutation frequency was less than $1.6 \times 10^{-10}$ cells in untreated cultures, but increased 38 fold to $6.2 \times 10^{-9}$ cells in cultures treated with $8 \mu \mathrm{M}$ wrwycr, and increased almost ninefold to $1.4 \times 10^{-9}$ cells in cultures treated with $16 \mu \mathrm{M}$ wrwycr. The reduced mutation frequency observed after $16 \mu \mathrm{M}$ wrwycr treatment compared with $8 \mu \mathrm{M}$ wrwycr treatment may be due to lesser fitness of the mutants and/or a reduced replication rate at the higher peptide concentration.

\section{ryhB overexpression is a cause of the wrwycr hypersensitivity of a $\Delta$ fur mutant and the enterobactin uptake mutants}

Because of the role Fur plays in regulation of the enterobactin uptake, we tested the sensitivity of the $\Delta f u r$ 

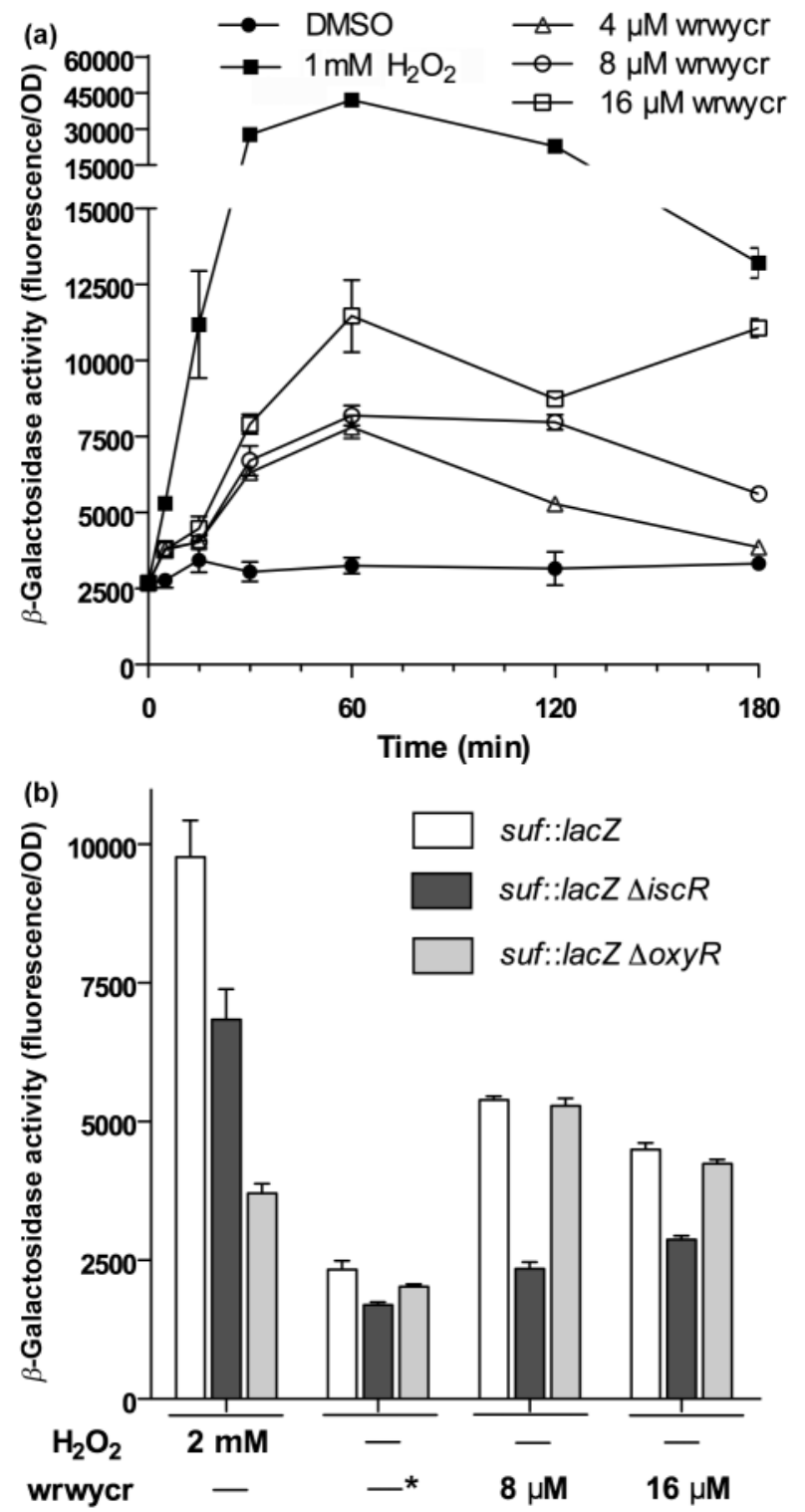

Fig. 4. Peptide wrwycr treatment activates the Suf operon through IscR. (a) Treatment with wrwycr induces a promoter fusion of psuf::lacZ; induction was followed over time using the $\beta$ galactosidase substrate MUG. Suf promoter activity expression takes longer to return to the level in untreated cultures with higher doses of wrwycr. (b) Suf operon activation by wrwycr is dependent upon the IscR activator. Three reporter strains, one lacking IscR, another lacking OxyR and their isogenic wild-type strain, were assayed after 60 min of wrwycr treatment. $(a, b) \mathrm{H}_{2} \mathrm{O}_{2}$ was used as a positive control to induce the Suf operon. DMSO, the solvent of the wrwycr was used as the negative control. The data are expressed as the mean $\pm S D$ of three independent colonies. Cells were grown and treated in MGCV medium. * Indicates DMSO treatment at $0.16 \%$.

mutant to wrwycr and found that it was hypersensitive to $20 \mu \mathrm{M}$ wrwycr (Fig. 5). When Fur is not bound to iron, as is typical in low-iron media conditions, it does not repress

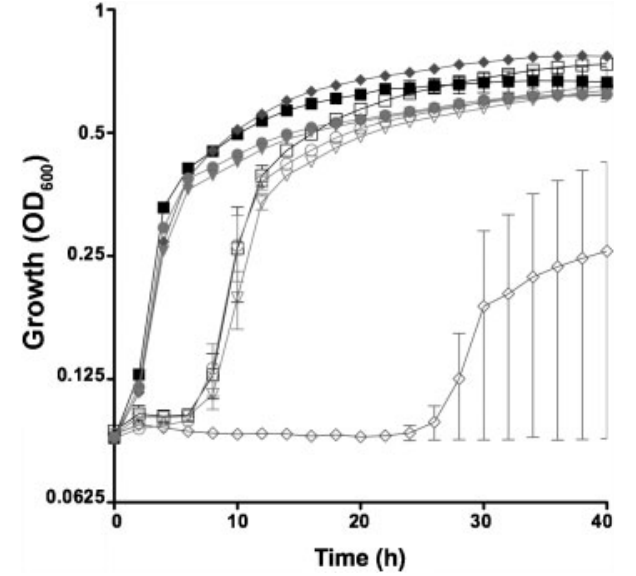

Fig. 5. The hypersensitivity to wrwycr of an $E$. coli $\Delta$ fur mutant is corrected by an added mutation, $\Delta r y h B$. Three individual colonies of each of MG1655 (G652, squares), $\Delta$ fur (EDT1566, diamonds), $\Delta r y h B$ (EDT1571, circles) and $\Delta$ fur $\Delta r y h B$ (EDT1712, triangles) were grown overnight in NGCV medium with no added iron, then diluted to $1 \%$ in fresh NGCV medium with $0.2 \%$ DMSO (closed symbols) or $20 \mu \mathrm{M}$ wrwycr (open symbols). The cultures (100 $\mu \mathrm{l}$ total) were grown in microtitre plates at $37{ }^{\circ} \mathrm{C}$. Each data point represents the mean \pm SEM $O_{600}$ of three individual cultures.

$r y h B$ transcription. In turn, the additional RyhB regulatory RNA molecules produced may decrease the expression of a gene that is important for wrwycr tolerance and/or increase expression of a wrwycr target during growth in the low-iron NGCV and MGCV media. In fact, the hypersensitivity of the $\Delta$ fur mutant was alleviated by the deletion of $\Delta r y h B$ (Fig. 5).

To further test the hypothesis that a RyhB-regulated gene is important for resistance to wrwycr, we introduced a $r y h B$ deletion into the $\Delta f e p B, \Delta f e p C, \Delta f e p D, \Delta f e p G$ and $\Delta f e s$ mutants to relieve repression of RyhB targets in low-iron media and assayed growth in the presence of wrwycr. As shown in Fig. 6, all the double mutants except the $\Delta f e p B$ $\Delta r y h B$ strain had near wild-type resistance to wrwycr, while the tested $\mathrm{RyhB}^{+}$single enterobactin uptake mutants showed their characteristic wrwycr hypersensitivity; in some experiments (data not shown), the $\Delta f e p B \Delta r y h B$ strain had near wild-type resistance to wrwycr, in contrast with the results shown in Fig. 6. We thus conclude that at least one RyhB-regulated gene is important for wrwycr resistance in the enterobactin-uptake mutants. However, deletion of $r y h B$ in the wild-type strain does not significantly increase its resistance to wrwycr, thus the altered expression of $r y h B$ due to its reduced repression by Fur in the iron-limited conditions following wrwycr treatment is not the sole cause of the sensitivity of the wild-type strain to wrwycr.

\section{DISCUSSION}

In this work, we showed that $E$. coli mutants defective in enterobactin biosynthesis or ferric enterobactin uptake 

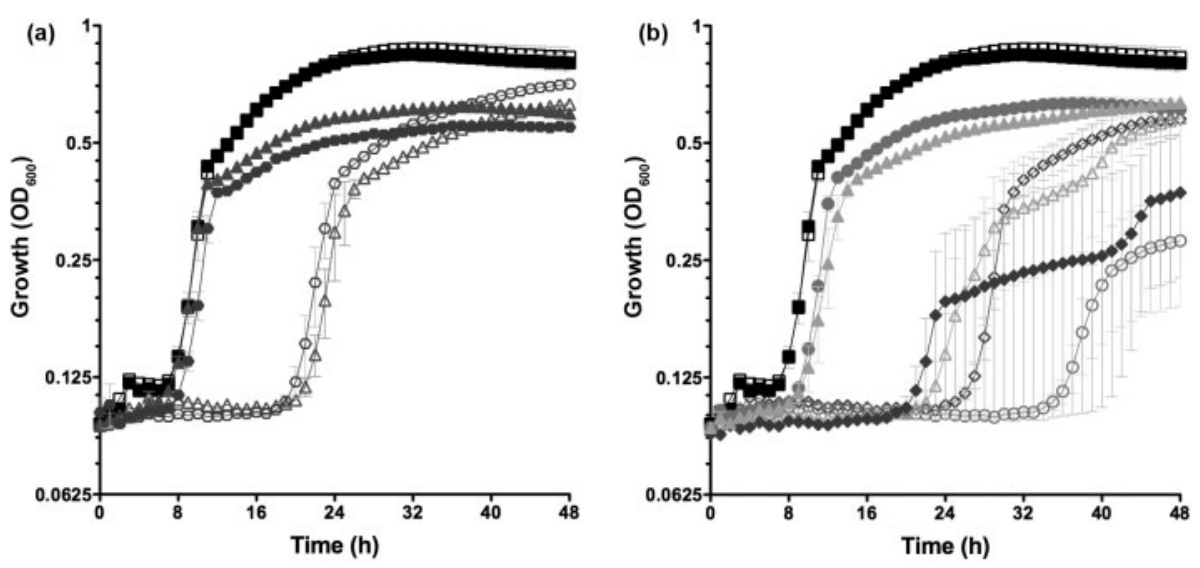

Fig. 6. Deletion of $r y h B$ reduces the hypersensitivity to wrwycr of the enterobactin uptake mutants. Open symbols indicate $\mathrm{RyhB}^{+}$strains and closed symbols indicate RyhB ${ }^{-}$strains. The mean $\pm \mathrm{SEM} \mathrm{OD}_{600}$ of three independent cultures of (a) E. coli MG1655 (G652, $\square), \Delta r y h B(E D T 1571, \mathbf{\square}), \Delta$ fepC (EDT1686, $\triangle), \Delta$ fepC $\Delta r y h B(E D T 1700, \Delta), \Delta f e p G(E D T 1687, \bigcirc)$ and $\Delta f e p G \Delta r y h B(E D T 1701, \bullet)$ or (b) E. coli MG1655 (G652, $\square), \Delta r y h B(E D T 1571, \square), \Delta f e p B(E D T 1343, \diamond), \Delta f e p B \Delta r y h B$ $(E D T 1683, \diamond), \Delta f e p D(E D T 1344, \bigcirc), \Delta f e p D \Delta r y h B(E D T 1684, \bullet), \Delta f e s(E D T 1345, \triangle)$ and $\Delta f e s \Delta r y h B(E D T 1685, \Delta)$ measured during growth in MGCV with $20 \mu \mathrm{M}$ wrwycr at $37{ }^{\circ} \mathrm{C}$. Growth measurements shown in (a) and (b) were recorded simultaneously but are presented in two panels for clarity. All strains grew equally well in DMSO (data not shown).

across the cytoplasmic membrane were hypersensitive to the synthetic antimicrobial peptide wrwycr. We infer from these results that the ability to take up ferric enterobactin or another compound that uses this same transport system is important for E. coli to withstand wrwycr treatment. Mutants lacking FepA or TonB, which are responsible for ferric enterobactin transport across the outer membrane, were not significantly hypersensitive to wrwycr. Our recent analysis of the E. coli proteome found that FepA protein levels decreased upon wrwycr treatment (J. E. Roston and other authors, unpublished data). Since FepA levels in the wild-type strain are reduced by wrwycr treatment, it is not surprising that the absence of FepA in the $\Delta f e p A$ mutant did not significantly increase sensitivity of $E$. coli to wrwycr. Ferric enterobactin may cross the outer membrane more easily in wrwycr-treated cells because the peptide increases the permeability of cells, as shown by greater fluorescence of lipophilic probes such as NPN (Jonnalagadda, 2009).

In support of ferric enterobactin limitation causing wrwycr hypersensitivity, we found that mutants lacking either EntB or EntC were also hypersensitive to wrwycr, although less so than some of the uptake mutants (perhaps the reason they were not identified in the original screen). The $\Delta e n t C$ strain was less hypersensitive to wrwycr than was the $\Delta e n t B$ strain, but a $\Delta m e n F \Delta e n t C$ strain was more sensitive than the $\Delta$ entC $\mathrm{MenF}^{+}$strain. This suggests that either the second isochorismate synthase in E. coli, MenF (Daruwala et al., 1996), can make isochorismate for the synthesis of enterobactin in the absence of $\mathrm{EntC}$ and/or menaquinone synthesis by MenF is itself important for resistance to wrwycr. Indeed, a $\triangle m e n F \mathrm{EntC}^{+}$strain was hypersensitive to wrwycr. The literature suggests that MenF cannot compensate for the lack of EntC for enterobactin synthesis unless the corresponding genes are overexpressed in trans, as most of the isochorismate synthesized by MenF and EntC is thought to be channelled to the next enzyme in their respective pathways, MenD or EntB (Buss et al., 2001). Sufficient isochorismate may escape the menaquinone pathway to make dihydroxybenzoate (and therefore enterobactin) in the absence of EntC in the semi-anaerobic conditions of the microtitre plate assays.

E. coli entS and tolC mutants are known to excrete little if any enterobactin but they do excrete monomer, dimer and trimer 2,3-dihydrobenzoylserine molecules (Bleuel et al., 2005; Furrer et al., 2002). These Fes-mediated enterobactin breakdown products are siderophores in their own right (in their iron-bound state they are taken back into the cell by the Fiu and Cir receptors) and thus entS and tolC mutants can achieve comparable growth to that of enterobactin-excreting strains (Hantke, 1990). As such, it is not surprising that the $\Delta e n t S$ mutant tested here was not hypersensitive to wrwycr. To explain the hypersensitivity of the $\Delta$ tolC mutant to wrwycr, we propose that TolC is responsible for efflux of wrwycr. Supporting this hypothesis, the $\Delta t o l C$ mutant had higher levels of cell-associated wrwycr as well as a reduced ratio of dimer to monomer wrwycr compared to MG1655 and the $\Delta f e p B$ mutant. In the $\Delta t o l C$ mutant, wrwycr may enter and remain in the cytoplasm long enough for a greater fraction to become reduced to the monomer form. Exactly how wrwycr enters bacteria to achieve the observed steadystate level of cell-associated wrwycr is unknown.

Why is the ability to harvest ferric enterobactin protective against wrwycr in a low-iron medium? We hypothesized that wrwycr treatment reduced bioavailable iron in the cell, necessitating increased iron uptake by the ferric enterobactin 
transporter. Supporting this hypothesis, the transcript level of the Fur: $\mathrm{Fe}^{2+}$-repressed genes entB and $f h u F$ increased and catalase activity decreased after $1.5 \mathrm{~h}$ wrwycr treatment. These results are consistent with wrwycr treatment reducing the amount of bioavailable iron. In contrast, direct measurement of chelatable iron in the cell indicated that wrwycr treatment actually increased chelatable iron, perhaps by damaging iron-sulfur cluster proteins, as has been proposed for other antibiotics (e.g. Gu \& Imlay, 2011; Kohanski et al., 2007). The induction of suf operon promoter activity by wrwycr treatment agrees with this idea, and implicates IscR (and not OxyR) as being necessary for this induction. A possible explanation for the apparent conflict between wrwycr's induction of genes that promote iron uptake and the cells' perceived shortage of iron is that the chelatable iron is not in fact available to bind to Fur or KatG and to rescue wrwycr-treated cells.

The increased iron uptake in the $\Delta$ fur mutant should logically have provided protection against wrwycr due to derepression of genes encoding iron uptake systems, and thus the $\Delta$ fur mutant should not have been hypersensitive to wrwycr, in conflict with our findings. However, perhaps the iron concentration of the test medium was not sufficient to counteract wrwycr challenge. Moreover, RyhB levels would have increased in the $\Delta$ fur mutant, decreasing expression of RyhB-repressed genes and increasing expression of RyhBactivated genes. Deletion of $r y h B$ mostly, but not completely, relieved the wrwycr hypersensitivity of the tested fep and fes mutants, indicating either that a RyhB-repressed function is critical for withstanding wrwycr treatment or that a RyhBactivated function somehow promotes the inhibitory effects of wrwycr. Deletion of the shiA gene, known to be activated by $\mathrm{RyhB}$, does not render either wild-type or $\Delta f e p B$ cells more sensitive than the $\mathrm{ShiA}^{+}$isogenic strains (data not shown). Future studies will be aimed at identifying the RyhB-targeted transcript(s) that affects wrwycr sensitivity. As the altered regulation by $\mathrm{RyhB}$ does not fully explain the hypersensitivity of the enterobactin uptake mutants, wrwycr-dependent iron limitation probably plays a role in addition to the altered iron regulation.

A surprising result is the discordant transcription response to wrwycr of entB and $f h u F$ in the $\Delta$ fur strain. One possibility for the observed differential response may be the additional positive regulation of entB by CRP-cAMP (Zhang et al., 2005). It is intriguing that in Salmonella, CRP-activated genes are induced about twofold in a $\Delta f u r$ strain by the presence of the iron chelating agent dipyridyl, similar to our observed induction of ent $B$ transcription in the presence of wrwycr (Campoy et al., 2002). The same study showed that the concentration of cAMP was higher in a $\Delta$ fur mutant compared with the wild-type in ironlimiting conditions in the presence of dipyridyl. We thus note the parallel between the effects of dipyridyl and wrwycr on ent $B$ expression (and perhaps, by inference, on cAMP levels) in the $\Delta$ fur mutant, although in the study by Campoy et al., the medium used was LB rather than the MHB media we used in our own qPCR experiments.
The observed Fur-regulon derepression in response to wrwycr indicates lower levels of bioavailable iron levels, but how does wrwycr treatment elicit this outcome? The most straightforward explanation would be that wrwycr chelates iron, albeit weakly. However, we have been unable to find any evidence for such an activity by using either a chrome azurol S colorimetric assay (Schwyn \& Neilands, 1987) or by monitoring the spectrophotometric or fluorometric profile of wrwycr in the presence of iron. We may not have found the appropriate conditions for testing binding of wrwycr to iron. Alternatively, instead of binding iron directly, wrwycr may indirectly sequester iron by binding a siderophore and causing its aggregation, for example, but we have no clues as to the identity of such a molecule. Our results could also formally be explained by wrwycr binding to Fur and KatG directly, competing for iron binding intracellularly (see below). We will test these hypotheses in the future.

It is unclear what the relationship is between the different activities of peptide wrwycr in interfering with DNA repair and accumulating intracellular DNA damage and the ironrestricting activities described here. At least two DNA glycosylases involved in base excision repair, endonuclease III (Nth) and MutY protein, and the DinG helicase, induced by DNA damage, contain $[4 \mathrm{Fe}-4 \mathrm{~S}]^{2+}$-type ironsulfur centres (Boal et al., 2005; Cunningham et al., 1989; Lukianova \& David, 2005; Ren et al., 2009). Impairment of the activity of MutY and Nth by wrwycr due to damage to their iron-sulfur centres may be one explanation for wrwycr-dependent increase in the mutation frequency in iron-limited conditions (NGCV media). Another explanation may be the presence of greater concentrations of intracellular iron after wrwycr treatment. Our best current hypothesis is that the effects of wrwycr on DNA repair (Gunderson et al., 2009) and on iron availability are independent, albeit potentially synergistic.

In summary, the antimicrobial properties of wrwycr are potentiated in low-iron conditions. Enterobactin has been thought not to be an important virulence factor because mammalian neutrophils produce siderocalin (lipocalin), which binds enterobactin in serum (Goetz et al., 2002). However, mammalian antimicrobial strategies include the export of iron from macrophages when they are infected with intracellular pathogens such as Salmonella, Mycobacteria and others (Forbes \& Gros, 2001; Nairz et al., 2007). Moreover, several of the effects of wrwycr on iron metabolism described herein, including the accumulation of chelatable but likely unavailable iron in cells, are independent of enterobactin, and thus wrwycr almost certainly puts a strain on all high-affinity iron uptake systems. This could make it or similar molecules particularly effective against pathogens in the low-iron conditions of their hosts.

\section{ACKNOWLEDGEMENTS}

The authors thank Elysa Brown, Ylva Sundstrom and R. Nathan Authement for their help in strain construction and performing some 
of the growth experiments, Paulina Gutierrez for measuring the mutation frequency, and Randy Reyes for performing the experiments shown in Fig. 4(b). We are grateful to Professor James Imlay and his student Yuanyuan Liu (University of Illinois, Urbana-Champaign) for hosting J.E.R. and assisting with the EPR analysis. We thank Professors James Imlay and Ian Booth (University of Aberdeen) for very helpful discussions, Ertan Ozyamak and Ian Booth for the protocol for the silicone oil/formic acid peptide extraction, Daniel Medina-Cleghorn for help with optimizing the silicone oil/formic acid protocol for our purposes, Jesse Woodson for critical reading of the manuscript, and Elizabeth Dinsdale for advice on statistics. This work was supported by National Institutes of Health RO1 grant AI058253 (awarded to A.M.S. and used to support S. S. O. in part) and GM052847 (A. M.S.). S. S. O. received additional support from a Ruth L. Kirschstein National Research Service Award postdoctoral fellowship from the National Institutes of Health (5 F32 GM77803).

\section{REFERENCES}

Baba, T., Ara, T., Hasegawa, M., Takai, Y., Okumura, Y., Baba, M., Datsenko, K. A., Tomita, M., Wanner, B. L. \& Mori, H. (2006). Construction of Escherichia coli K-12 in-frame, single-gene knockout mutants: the Keio collection. Mol Syst Biol 2 doi:10.1038/msb4100050.

Bagg, A. \& Neilands, J. B. (1987). Ferric uptake regulation protein acts as a repressor, employing iron (II) as a cofactor to bind the operator of an iron transport operon in Escherichia coli. Biochemistry 26, 5471-5477.

Bleuel, C., Grosse, C., Taudte, N., Scherer, J., Wesenberg, D., Krauss, G. J., Nies, D. H. \& Grass, G. (2005). TolC is involved in enterobactin efflux across the outer membrane of Escherichia coli. J Bacteriol 187, 6701-6707.

Boal, A. K., Yavin, E., Lukianova, O. A., O'Shea, V. L., David, S. S. \& Barton, J. K. (2005). DNA-bound redox activity of DNA repair glycosylases containing [4Fe-4S] clusters. Biochemistry 44, 83978407.

Boldt, J. L., Pinilla, C. \& Segall, A. M. (2004). Reversible inhibitors of lambda integrase-mediated recombination efficiently trap Holliday junction intermediates and form the basis of a novel assay for junction resolution. J Biol Chem 279, 3472-3483.

Brickman, T. J. \& Mclntosh, M. A. (1992). Overexpression and purification of ferric enterobactin esterase from Escherichia coli. Demonstration of enzymatic hydrolysis of enterobactin and its iron complex. J Biol Chem 267, 12350-12355.

Buss, K., Müller, R., Dahm, C., Gaitatzis, N., Skrzypczak-Pietraszek, E., Lohmann, S., Gassen, M. \& Leistner, E. (2001). Clustering of isochorismate synthase genes menF and ent $C$ and channeling of isochorismate in Escherichia coli. Biochim Biophys Acta 1522, 151-157.

Campoy, S., Jara, M., Busquets, N., de Rozas, A. M., Badiola, I. \& Barbé, J. (2002). Intracellular cyclic AMP concentration is decreased in Salmonella typhimurium fur mutants. Microbiology 148, 1039-1048.

Cherepanov, P. P. \& Wackernagel, W. (1995). Gene disruption in Escherichia coli: $\mathrm{Tc}^{\mathrm{R}}$ and $\mathrm{Km}^{\mathrm{R}}$ cassettes with the option of Flpcatalyzed excision of the antibiotic-resistance determinant. Gene 158, 9-14.

Cunningham, R. P., Asahara, H., Bank, J. F., Scholes, C. P., Salerno, J. C., Surerus, K., Münck, E., McCracken, J., Peisach, J. \& Emptage, M. H. (1989). Endonuclease III is an iron-sulfur protein. Biochemistry 28, 4450-4455.

Daruwala, R., Kwon, O., Meganathan, R. \& Hudspeth, M. E. (1996). A new isochorismate synthase specifically involved in menaquinone (vitamin K2) biosynthesis encoded by the menF gene. FEMS Microbiol Lett 140, 159-163.
Davis, R. W., Botstein, D. \& Roth, J. R. (1980). Advanced Bacterial Genetics: a Manual for Genetic Engineering. Cold Spring Harbor, NY: Cold Spring Harbor Laboratory.

Fenton, H. (1894). Oxidation of tartaric acid in presence of iron. J Chem Soc Trans 65, 899.

Forbes, J. R. \& Gros, P. (2001). Divalent-metal transport by NRAMP proteins at the interface of host-pathogen interactions. Trends Microbiol 9, 397-403.

Furrer, J. L., Sanders, D. N., Hook-Barnard, I. G. \& McIntosh, M. A. (2002). Export of the siderophore enterobactin in Escherichia coli: involvement of a $43 \mathrm{kDa}$ membrane exporter. Mol Microbiol 44, 1225-1234.

Goetz, D. H., Holmes, M. A., Borregaard, N., Bluhm, M. E., Raymond, K. N. \& Strong, R. K. (2002). The neutrophil lipocalin NGAL is a bacteriostatic agent that interferes with siderophore-mediated iron acquisition. Mol Cell 10, 1033-1043.

Gu, M. \& Imlay, J. A. (2011). The SoxRS response of Escherichia coli is directly activated by redox-cycling drugs rather than by superoxide. Mol Microbiol 79, 1136-1150.

Guerinot, M. L. (1994). Microbial iron transport. Annu Rev Microbiol 48, 743-772.

Gunderson, C. W. \& Segall, A. M. (2006). DNA repair, a novel antibacterial target: Holliday junction-trapping peptides induce DNA damage and chromosome segregation defects. Mol Microbiol 59, 1129-1148.

Gunderson, C. W., Boldt, J. L., Authement, R. N. \& Segall, A. M. (2009). Peptide wrwycr inhibits the excision of several prophages and traps Holliday junctions inside bacteria. J Bacteriol 191, 2169-2176.

Haber, F. \& Weiss, J. (1932). Über die Katalyse des Hydroperoxydes. Naturwiss 20, 948-950.

Haber, F. \& Weiss, J. (1934). The catalytic decomposition of hydrogen peroxide by iron salts. Proc R Soc Lond A Math Phys Sci 147, 332-351.

Haber, F. \& Willstätter, R. (1931). Unpaarigkeit und Radikalketten im Reaktionsmechanismus organischer und enzymatischer Vorgänge. Chem Ber 64, 2844-2856.

Halliwell, B. \& Gutteridge, J. M. (1984). Oxygen toxicity, oxygen radicals, transition metals and disease. Biochem J 219, 1-14.

Hantke, K. (1990). Dihydroxybenzoylserine - a siderophore for $E$. coli. FEMS Microbiol Lett 55, 5-8.

Hubbard, J. A., Lewandowska, K. B., Hughes, M. N. \& Poole, R. K. (1986). Effects of iron-limitation of Escherichia coli on growth, the respiratory chains and gallium uptake. Arch Microbiol 146, 8086.

Imlay, J. A., Chin, S. M. \& Linn, S. (1988). Toxic DNA damage by hydrogen peroxide through the Fenton reaction in vivo and in vitro. Science 240, 640-642.

Jonnalagadda, U. M. (2009). The effects of antibacterial peptides on bacterial and membrane integrity and their mechanism of cellular entry. Master's thesis, San Diego State University, San Diego, USA.

Kepple, K. V., Boldt, J. L. \& Segall, A. M. (2005). Holliday junctionbinding peptides inhibit distinct junction-processing enzymes. Proc Natl Acad Sci U S A 102, 6867-6872.

Kohanski, M. A., Dwyer, D. J., Hayete, B., Lawrence, C. A. \& Collins, J. J. (2007). A common mechanism of cellular death induced by bactericidal antibiotics. Cell 130, 797-810.

Koronakis, V. (2003). TolC - the bacterial exit duct for proteins and drugs. FEBS Lett 555, 66-71.

Koronakis, V., Eswaran, J. \& Hughes, C. (2004). Structure and function of TolC: the bacterial exit duct for proteins and drugs. Annu Rev Biochem 73, 467-489. 
Li, Y. \& Schellhorn, H. E. (2007). Rapid kinetic microassay for catalase activity. J Biomol Tech 18, 185-187.

Lino, M., Kus, J. V., Tran, S. L., Naqvi, Z., Binnington, B., Goodman, S. D., Segall, A. M. \& Foster, D. B. (2011). A novel antimicrobial peptide significantly enhances acid-induced killing of Shiga toxinproducing Escherichia coli $\mathrm{O} 157$ and non-O157 serotypes. Microbiology 157, 1768-1775.

Lukianova, O. A. \& David, S. S. (2005). A role for iron-sulfur clusters in DNA repair. Curr Opin Chem Biol 9, 145-151.

Massé, E. \& Gottesman, S. (2002). A small RNA regulates the expression of genes involved in iron metabolism in Escherichia coli. Proc Natl Acad Sci U S A 99, 4620-4625.

Massé, E., Escorcia, F. E. \& Gottesman, S. (2003). Coupled degradation of a small regulatory RNA and its mRNA targets in Escherichia coli. Genes Dev 17, 2374-2383.

Massé, E., Vanderpool, C. K. \& Gottesman, S. (2005). Effect of RyhB small RNA on global iron use in Escherichia coli. J Bacteriol 187, 69626971.

Miller, J. H. (1972). Experiments in Molecular Genetics. Cold Spring Harbor, NY: Cold Spring Harbor Laboratory.

Nairz, M., Theurl, I., Ludwiczek, S., Theurl, M., Mair, S. M., Fritsche, G. \& Weiss, G. (2007). The co-ordinated regulation of iron homeostasis in murine macrophages limits the availability of iron for intracellular Salmonella typhimurium. Cell Microbiol 9, 2126-2140.

Neidhardt, F. C., Bloch, P. L. \& Smith, D. F. (1974). Culture medium for enterobacteria. J Bacteriol 119, 736-747.

Neilands, J. B. (1981). Iron absorption and transport in microorganisms. Annu Rev Nutr 1, 27-46.

Outten, F. W., Djaman, O. \& Storz, G. (2004). A suf operon requirement for $\mathrm{Fe}-\mathrm{S}$ cluster assembly during iron starvation in Escherichia coli. Mol Microbiol 52, 861-872.
Prévost, K., Salvail, H., Desnoyers, G., Jacques, J. F., Phaneuf, E. \& Massé, E. (2007). The small RNA RyhB activates the translation of shiA mRNA encoding a permease of shikimate, a compound involved in siderophore synthesis. Mol Microbiol 64, 1260-1273.

Raymond, K. N., Dertz, E. A. \& Kim, S. S. (2003). Enterobactin: an archetype for microbial iron transport. Proc Natl Acad Sci U S A 100, 3584-3588.

Ren, B., Duan, X. \& Ding, H. (2009). Redox control of the DNA damage-inducible protein DinG helicase activity via its iron-sulfur cluster. J Biol Chem 284, 4829-4835.

Schwyn, B. \& Neilands, J. B. (1987). Universal chemical assay for the detection and determination of siderophores. Anal Biochem 160, 4756.

Su, L. Y., Willner, D. L. \& Segall, A. M. (2010). An antimicrobial peptide that targets DNA repair intermediates in vitro inhibits Salmonella growth within murine macrophages. Antimicrob Agents Chemother 54, 1888-1899.

Vassinova, N. \& Kozyrev, D. (2000). A method for direct cloning of Fur-regulated genes: identification of seven new Fur-regulated loci in Escherichia coli. Microbiology 146, 3171-3182.

Woodmansee, A. N. \& Imlay, J. A. (2002). Quantitation of intracellular free iron by electron paramagnetic resonance spectroscopy. Methods Enzymol 349, 3-9.

Yeo, W. S., Lee, J. H., Lee, K. C. \& Roe, J. H. (2006). IscR acts as an activator in response to oxidative stress for the suf operon encoding Fe-S assembly proteins. Mol Microbiol 61, 206-218.

Zhang, Z., Gosset, G., Barabote, R., Gonzalez, C. S., Cuevas, W. A. \& Saier, M. H., Jr (2005). Functional interactions between the carbon and iron utilization regulators, Crp and Fur, in Escherichia coli. J Bacteriol 187, 980-990.

Edited by: A. J. O’Neill 